\title{
Asymmetric Limit Cycles in an Industrially Applied Controlled Resonant Converter
}

\author{
Anders Hultgren*, Jan Melin**, and Per Ranstad*** \\ *Blekinge Institute of Technology, Sweden (Tel: +46 7094028 53, e-mail: anders.hultgren@bth.se) \\ **University of Kalmar, Dep. of Natural Sciences, Sweden (e-mail: jan.melin@hik.se) \\ ***Alstom Power, PSP, Environmental R\&D, Sweden, (e-mail: per.ranstad@power.alstom.com)
}

\begin{abstract}
An industrially applied LCC power converter is modelled as a hybrid system. It is found that the hybrid system given a parameter set up and different initial conditions has three different limit cycles, one unstable and two asymmetric stable limit cycles. Effects of the asymmetric limit cycles are considered.
\end{abstract}

Keywords: Modelling, analysis, simulation, control, hybrid, limit cycles, resonant power converters.

\section{INTRODUCTION}

DC/DC converters are today mainly controlled by switching devices causing abrupt changes in the converter dynamics. Modelling the switch devices as ideal, the controlled converters fall within the group of hybrid dynamical systems, see van der Schaft and Schumacher (2000) and Orlov (2009). The variety of topology of DC/DC converters is huge. In this report the control of an industrially applied resonant converter is discussed. Resonant converters have possibly many good properties, i.e. switching losses.

There are several suggestions of how to design a switched controller for resonant converters. Oruganti and Lee (1985) presented a set of switched state feedback controllers and since then several authors have addressed the design and analysis question of resonant converter controllers. See for instance Rossetto (1996) and Sng et al (2006) and Hultgren et al (2002). Some of the control laws suggested generate piecewise systems, possibly piecewise linear systems and some of others generate a more general subclass of hybrid systems.

Careful analysis of switched resonant converters, also when modelled with only two continuous states and as a piecewise linear system, can show that the possible solution set can be very rich. See for instance Melin et al (2003) and Melin and Hultgren (2006). When modelling with three continuous states the solution set will increase even more, see Melin et al (2008). Higher order models and derivation of an observer for the converter model can be found in Hultgren and Lenells (2004a, b). Methods for computation of limit cycles in hybrid systems can be found in and Flieller and Louis (2006).

The industrially applied resonant converter, described in this report, is modeled as a series parallel, LCC, converter, see the circuit diagram in Fig. 1. The suggested controller is a switched feedback controller, derived to have high robustness for load disturbances. The solution set appears to be richer than expected and is together with the industrial implications addressed as the main topics in this report.

The series part of the converter consists of the capacitance, C, and the inductance, $\mathrm{L}$. The parallel capacitance, $\mathrm{C}_{\mathrm{w}}$, models parasitic effects in the transformer, TR, and has the size of about $15 \%$ of the series capacitance, $\mathrm{C}$. The resistance $\mathrm{R}$ is neglected in the analysis. The controlled output of the converter is the current, $\mathrm{I}_{0}$, to the load capacitance, $\mathrm{C}_{0}$, on the secondary side of the transformer. The resonant converter is applied in a high voltage equipment. The capacitive load and high transformer ratio will give a comparatively large effective load capacitance, making the load voltage, $\mathrm{U}_{0}$, only slowly varying. Hence, the load voltage is modeled as a constant voltage source, with the value $\mathrm{U}_{0}$, , on the primary side of the transformer. The converter is supplied by the voltage, E, and a full transistor bridge is controlling the voltage across the resonant circuit between junctions $\mathrm{A}$ and $\mathrm{B}, u_{A B}$. Assuming ideal transistor switches, the control signal can then be taken out of a set of three levels, $u_{A B} \in\{-E, 0, E\}$. The resonance frequency is $20 \mathrm{kHz}$ and the controller is running the converter in a limit cycle above the resonance frequency, generating low switch-on losses in the transistors.

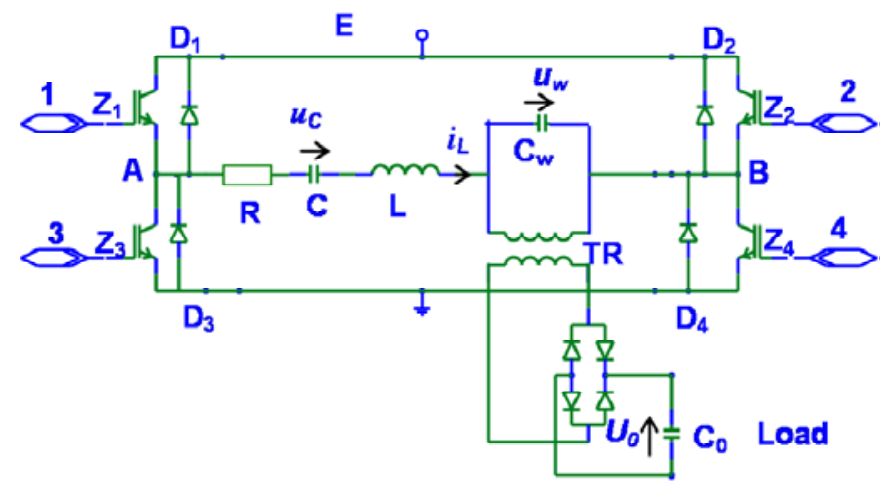

Fig. 1. The resonant converter used in an industrial application

In spite of the large load capacitance there are frequently load voltage disturbances in the industrial application that the controller has to cope with. The suggested controller is derived with the aim of robustness for load voltage disturbances. The controller is implemented in FPGA technology, implying that intensive calculations can be performed and that the sampling interval can be short. 


\section{THE CONVERTER MODEL}

\subsection{Choice of Controller}

Choosing the two most important states as the normalized series capacitance voltage, $\mathrm{u}_{\mathrm{CN}}$, and inductance current, $\mathrm{i}_{\mathrm{LN}}$, which implies easier calculations, the resonant converter is given by the second order system

$$
\begin{aligned}
& \left(\begin{array}{l}
\dot{x} \\
\dot{y}
\end{array}\right)=\left(\begin{array}{cc}
0 & \frac{1}{\sqrt{L C}} \\
\frac{-1}{\sqrt{L C}} & 0
\end{array}\right)\left(\begin{array}{l}
x \\
y
\end{array}\right)+\left(\begin{array}{c}
0 \\
\frac{s-U_{0 N} \cdot \operatorname{sign}(y)}{\sqrt{L C}}
\end{array}\right) \\
& \text { where }\left(\begin{array}{ll}
\mathrm{x} & y
\end{array}\right)^{T}=\left(\begin{array}{ll}
u_{C N} & i_{L N}
\end{array}\right)^{T}=\left(\begin{array}{ll}
\frac{u_{C}}{E} & \frac{i_{L}}{E} \sqrt{\frac{L}{C}}
\end{array}\right)^{T} \\
& \text { and } U_{0 N}=\frac{U_{0}{ }^{\prime}}{E}, s=\frac{u_{A B}}{E} \in\{-1,0,1\}
\end{aligned}
$$

The system is run in a limit cycle by choosing $s=1$, each time the current $i_{L}$ change to positive sign and $s=-1$ each time current $i_{L}$ change to negative sign. By means of choosing $s=$ 0 at a certain state position $(x, y)$ the size of the limit cycle can be adjusted to stationary fit the chosen desired load current $\mathrm{I}_{0 \mathrm{~N}}$ and the load voltage $\mathrm{U}_{0 \mathrm{~N}}$. In Fig. 2 the chosen switch curves for a set of load currents can be seen as the curves through the stars. A star represents a stationary switch point for a certain (I0N, U0N). It turns out that a switch curve can quite accurately be described by an ellipse. The ellipse radii are given as a function of the load current setpoint, $I_{0 N}$, in the Table 1.

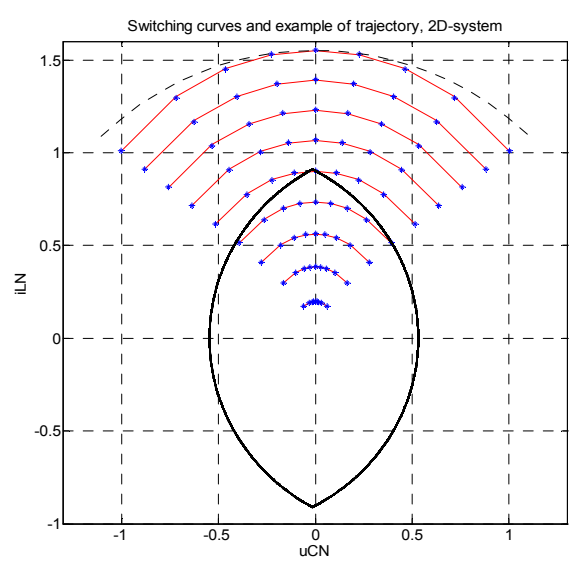

Fig. 2. Ellipses for different load current are shown in the upper half plane. The stars on a certain ellipse arc indicate the switch point (intersection point with the ellipse) for a certain load voltage. An example of stationary limit cycle for the case $I_{0 N}=U_{0 N}=0.5$ is shown.

\subsection{Modelling the Closed Third Order System}

The chosen controller is evaluated for the third order model of the converter, including the state, $z$, as the normalized $u_{w}=u_{w} / E$. The closed system will be given by a hybrid dynamic model with three continuous states and two logic states.
Fig. 3 shows a state graph for the logic states, I and II, for the controlled system is shown. When the logic state I is true, the normalized system is given by the piecewise linear system

$$
\left(\begin{array}{c}
\dot{x} \\
\dot{y} \\
\dot{z}
\end{array}\right)=\left(\begin{array}{ccc}
0 & \frac{1}{\sqrt{L C}} & 0 \\
\frac{-1}{\sqrt{L C}} & 0 & \frac{-1}{\sqrt{L C}} \\
0 & \Gamma & 0
\end{array}\right)\left(\begin{array}{l}
x \\
y \\
z
\end{array}\right)+\left(\begin{array}{c}
0 \\
\frac{1}{\sqrt{L C}} \\
0
\end{array}\right) \cdot \operatorname{sign}(y)
$$

where

$$
\Gamma=\left\{\begin{aligned}
0, & \text { when } z \geq U_{0 N} \text { and } \mathrm{y}>0 \text { or } z \leq-U_{0 N} \text { and } \mathrm{y}<0 \\
\frac{\sqrt{C}}{C_{w} \sqrt{L}}, & \text { elsewhere }
\end{aligned}\right.
$$

When the logic state II is true, the system is given by the piecewise linear system

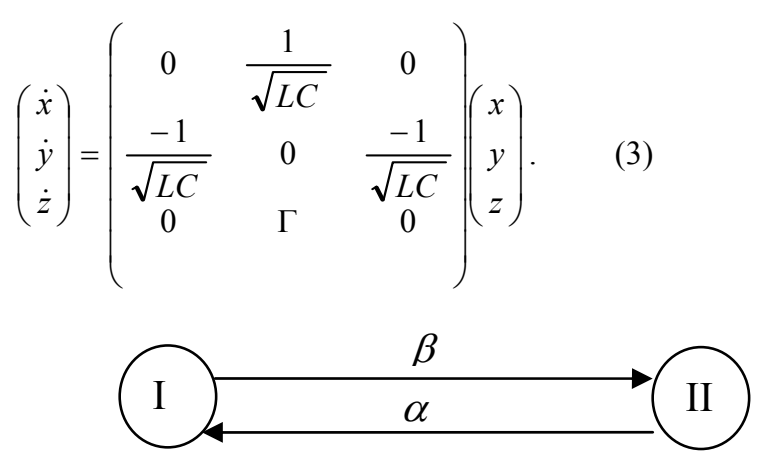

Fig. 3. State graph for the controlled converter

The transition condition $a$, shown in Fig 3, is fulfilled if the state trajectory passes the $y=0$ plane and the transition condition $\beta$ is fulfilled if the state trajectory projected onto the xy-plane hits the chosen ellipse in the xy-plane. In the third order system, xyz, the elliptic switch curves will act as an elliptic cylinder. Fig. 4 shows the elliptic cylinder surface for a certain choice of $\mathrm{I}_{0 \mathrm{~N}}$ is indicated by three ellipses.

Table 1. Radii a and $\mathrm{b}$ for different ellipses, $\frac{\mathrm{x}^{2}}{a^{2}}+\frac{y^{2}}{b^{2}}=1$ corresponding to different load currents, $\mathrm{I}_{0 \mathrm{~N}}$.

\begin{tabular}{|c|c|c|}
\hline $\mathrm{I}_{0 \mathrm{~N}}$ & Radie a (x) & Radie b (y) \\
\hline 0.1000 & 0.1238 & 0.1973 \\
\hline 0.2000 & 0.2611 & 0.3842 \\
\hline 0.3000 & 0.4057 & 0.5616 \\
\hline 0.4000 & 0.5542 & 0.7329 \\
\hline 0.5000 & 0.7051 & 0.9003 \\
\hline 0.6000 & 0.8575 & 1.0652 \\
\hline 0.7000 & 1.0109 & 1.2284 \\
\hline 0.8000 & 1.1650 & 1.3903 \\
\hline 0.9000 & 1.3196 & 1.5514 \\
\hline
\end{tabular}

\subsection{Following Trajectories of the System}

In order to easier follow trajectories in the system the over all valid equations (2) and (3) is reformulated for different regions of the state space. 
It is clear by (2) that the trajectories of the system always reside in between or onto the planes $\mathrm{z}=U_{0 \mathrm{~N}}$ and $\mathrm{z}=-U_{0 \mathrm{~N}}$ making $|z| \leq U_{0 \mathrm{~N}}$. The system can, when the trajectory resides in between these planes, be expressed by the following three-dimensional hybrid system:

$$
\left(\begin{array}{c}
\dot{x} \\
\dot{y} \\
\dot{z}
\end{array}\right)=\left(\begin{array}{ccc}
0 & \frac{1}{\sqrt{L C}} & 0 \\
\frac{-1}{\sqrt{L C}} & 0 & \frac{-1}{\sqrt{L C}} \\
0 & \frac{\sqrt{C}}{C_{w} \sqrt{L}} & 0
\end{array}\right)\left(\begin{array}{l}
x \\
y \\
z
\end{array}\right)+\left(\begin{array}{c}
0 \\
\frac{s}{\sqrt{L C}} \\
0
\end{array}\right)
$$

where $s$ is defined in (1). When the trajectory resides onto the limiting planes $z=U_{0 \mathrm{~N}}$ and $\mathrm{z}=-U_{0 \mathrm{~N}}$ the system is given by a two-dimensional system defined in (1). Considering the case when the trajectory resides between the two limiting planes and combining the first and the third equation of the three-dimensional model (4) we have $z=\frac{C}{C_{w}} \cdot x+D$, where $D$ is an integration constant. Putting this in the second equation we obtain the following twodimensional system projected onto the $x y$-plane: $\left(\begin{array}{l}\dot{x} \\ \dot{y}\end{array}\right)=\left(\begin{array}{cc}0 & 0 \\ 1+\frac{C}{C_{w}} & 0\end{array}\right) \cdot\left(\begin{array}{l}x \\ y\end{array}\right)+\left(\begin{array}{c}0 \\ \frac{s-D}{\sqrt{L C}}\end{array}\right)$.

A compact elliptic cylinder is defined by $\Omega$ : $\frac{x^{2}}{a^{2}}+\frac{y^{2}}{b^{2}} \leq 1$, where the half axis $a$ and $b$ are defined in section 1 above and given in Table $1 . \Omega$ is divided into the two sub regions $\Omega+: y>0$ and $\Omega-: y<0$. Whenever a trajectory moves from $\Omega+$ to $\Omega$ - then $s=-1$, from $\Omega$ - to $\Omega+$ then $s=1$ and after every impact with the ellipse $\frac{x^{2}}{a^{2}}+\frac{y^{2}}{b^{2}}=1$ then $s=0$. The switching surfaces are the plane $y=0$, the elliptic cylinder surface, the top of the cylinder $z=U_{0 \mathrm{~N}}$ and the bottom of the cylinder $z=-U_{0 \mathrm{~N}}$. The fixed points are the straight line $x+z=s$ in the plane $y=0$ and the eigenvalues are $\mu=0$ and $\mu= \pm \frac{i}{L} \cdot\left(\frac{1}{C}+\frac{1}{C_{w}}\right)$.

\section{THE SYSTEM TRAJECTORIES}

In this section some properties of the trajectories and possible limit cycles are derived.

Conjecture. A trajectory that starts in the cylinder stays in the cylinder.

Consider the top of the cylinder, $z=U_{0 N}$, in the upper half plane, $y>0$. Immediately after an intersection between a trajectory and the ellipse at a point $\left(x_{0}, y_{0}\right)$ where $-a<x_{0}<$ $a$ and $0<y_{0}<b$ we have $s=0$ and from equation (5) we obtain the system: $\left(\begin{array}{c}\dot{x} \\ \dot{y}\end{array}\right)=\left(\begin{array}{c}\frac{y}{\sqrt{L C}} \\ -\frac{x+U_{0 N}}{\sqrt{L C}}\end{array}\right)$. The slope of the tangent at the intersection point is $\left|\frac{d y}{d x}\right|=-\frac{x_{0}+U_{0 N}}{y_{0}}$. The slope of the tangent of the ellipse is $\left|\frac{d y}{d x}\right|=-\frac{b^{2}}{a^{2}} \cdot \frac{x_{0}}{y_{0}}$. A sufficient condition for the trajectory to stay in the cylinder is $\left|\left(\frac{d y}{d x}\right)_{\text {trajectory }}\right|>\left|\left(\frac{d y}{d x}\right)_{\text {ellipse }}\right|$, this leads to the inequality $x_{0}<\frac{U_{0 N}}{\left(\frac{b}{a}\right)^{2}-1}$. For each $I_{0 N}=0.1,0.2, \ldots, 0.9$ and $U_{0 N}=0.1,0.2, \ldots, 0.9$ simulations of the limit cycle show that the inequality is satisfied. A corresponding investigation has been done for limit cycles intersecting the cylinder between the top and the bottom as well as at the bottom of the cylinder and the inequality is still satisfied. This affirms the conjecture.

The section will now continue by calculating trajectories and derive two lemmas.

1) When a trajectory is located in the $\operatorname{region}|z|<$ $U_{0 N}$ between the top and the bottom of the cylinder, according to the first and third equation of the system a family of planes $z=\frac{C}{C_{w}} \cdot x+D$ are generated where the trajectory is located. For each starting point of the trajectory the plane is unique and the constant $D$ can be calculated. Using the equation of a plane and projecting the system onto the $x y$-plane and the trajectory is given by the equation $\frac{d y}{d x}=-\frac{\left(1+\frac{C}{C_{w}}\right) \cdot x-s+D}{y} \quad$ with the solution $\frac{y^{2}}{2}=-\left(1+\frac{C}{C_{w}}\right)$. $\frac{x^{2}}{2}+(s-D) \cdot x+F$. The integration constant $F$ is depending on the initial value.

2) At the bottom $z=-U_{0 N}$ of the cylinder the projected equation becomes $\frac{d y}{d x}=-\frac{x-U_{0 N}-s}{y}$ with the solution $\frac{y^{2}}{2}=-\frac{x^{2}}{2}+\left(U_{0 N}+s\right) \cdot x+F$.

3) The corresponding solution at the top $z=U_{0 N}$ becomes $\frac{y^{2}}{2}=-\frac{x^{2}}{2}+\left(s-U_{0 N}\right) \cdot x+F$.

Considering the region entirely inside the cylinder $\Omega$ and chosing a starting point $P_{0}:\left(x_{0}, 0, z_{0}\right)$, the trajectory follows the plane $z=\frac{C}{C_{w}} \cdot x+D$ where $D=z_{0}-\frac{C}{C_{w}} \cdot x_{0}$ and enters the region $\Omega$ - and $s=-1$. The solution is given by $y^{2}=$ $-\left(1+\frac{C}{C_{w}}\right) x^{2}-2(D+1) x+\left(1+\frac{C}{C_{w}}\right) x_{0}^{2}+2(D+1) x_{0}$.

The trajectory then intersects the switching surface $y=0$ at $x_{1}=-x_{0}-\frac{2(D+1)}{1+\frac{C}{C_{W}}}$. The trajectory enters $\Omega+$ and follows a new plane upwards. Considering $s=1$ and using the initial value $x=x_{1}$ when $y=0$, the solution is given by $y^{2}=$ $-\left(1+\frac{C}{C_{w}}\right) x^{2}-2(D-1) x+\left(1+\frac{C}{C_{w}}\right) x_{0}^{2}+2(D+3) x_{0}+$ $\frac{8(D+1)}{1+\frac{C}{C_{w}}}$. The trajectory returns to the switching surface $y=$ 0 at $x_{2}=x_{0}+\frac{4}{1+\frac{C}{C_{w}}}$ which implies $x_{2} \neq x_{0}$. The following Lemma is proved. 
Lemma 1. The system possesses no limit cycles entirely inside the cylinder.

Now we consider the plane $z=\frac{C}{C_{w}} \cdot x$ through origin. Any closed orbit, symmetric with respect to origin, has to be partly located in this plane. According to Lemma 1 a trajectory have to pass through the point $P_{0}:\left(\frac{C_{W}}{C} U_{0 N}, 0, U_{0 N}\right)$ at the top of the cylinder. The trajectory follows the plane and intersects the bottom of the cylinder at $x_{1}=-\frac{C_{w}}{C} \cdot U_{0 N}$. According to the first equation of the system $x$ is decreasing in this region, this implies that the trajectory must intersect the switching plane $y=0$ at $x_{2}<x_{1}$. The following Lemma is proved.

Lemma 2 . The system possesses no symmetric limit cycles with respect to origin.

\section{NUMERICAL CALCULATIONS}

Simulations indicate that the system possesses three different limit cycles in the compact cylinder $\Omega$. One of them is unstable and centrally located in the cylinder. The other two are asymptotically stable and located to the left respectively to the right, see Fig. 4 .

To confirm the indicated limit cycle properties the following derivations are made.

\subsection{The Centrally Located Closed Trajectory}

For a starting point at the top of the cylinder $P_{0}:\left(x_{0}, 0, U_{0 N}\right)$, the trajectory enters $\Omega$ - and follows the plane $z=\frac{C}{C_{w}} \cdot x+$ $U_{0 N}-\frac{C}{C_{w}} \cdot x_{0} \quad$ to the bottom with intersection at $P_{1}:\left(x_{1}, y_{1},-U_{0 N}\right)$. The trajectory is now located in the plane $z=-U_{0}$ and moves to the left until it intersects the ellipse at $P_{2}:\left(x_{2}, y_{2},-U_{0 N}\right)$. The trajectory continues to the left inside the cylinder until it intersects the switching surface $y=0$ at $P_{3}:\left(x_{3}, 0,-U_{0 N}\right)$. The trajectory then enters $\Omega+$ and follows the plane $z=\frac{C}{C_{w}} \cdot x-U_{0 N}-\frac{C}{C_{w}} \cdot x_{3}$ until it reaches the top at $P_{4}:\left(x_{4}, y_{4}, U_{0 N}\right)$. It moves to the right at the top $z=U_{0 N}$ and intersects the ellipse again at $P_{5}:\left(x_{5}, y_{5}, U_{0 N}\right)$. Finally the trajectory returns to the switching surface $y=0$ at the top with intersection at $P_{6}:\left(x_{6}, 0, U_{0 N}\right)$. Using appropriate trajectory equations introduced in section 3 with corresponding $s$, all switching points $P_{i}$ can be expressed in terms of the starting point. Then the equation $x_{6}=x_{0}$ can be solved with respect to $x_{0}$. Using the parameter values $C=2 \cdot 10^{-6}, \quad C_{w}=0.2 \cdot 10^{-6}$ and $U_{0 N}=0.5, x_{0}=$ 0.5268 implying that there is a closed trajectory through the points $P_{0}:(0.5268,0,0.5), P_{1}:(0.4268,-0.5435,-0.5)$, $P_{2}:(0.1000,-0.8912,-0.5) \quad P_{3}:(-0.4769,0,-0.5)$, $P_{4}:(-0.3769,0.5342,0.5), \quad P_{5}: \quad(0.0063,0.9003,0.5) \quad$ and finally back to $P_{6}=P_{0}$. Choosing $x_{0}=0.6$, the trajectory returns to $x_{6}=0.3886$. This indicates that this closed trajectory is unstable.

\subsection{The Limit Cycle Located to the left in the Cylinder}

Choosing a starting point $P_{0}$ at the top of the cylinder in the switching plane $y=0$, the trajectory basically follows the same track as the one in described in section 4.1. It intersects the bottom at $P_{1}$ then the ellipse at $P_{2}$, the switching plane at $P_{3}$. On its way up it differs from the previous trajectory, this one intersect the cylinder shell at $P_{4}$. It continues to the top to the point $P_{5}$ and finally returns the switching surface at $P_{6}$. Using the same parameter values as in 4.1 there is a closed trajectory through the points $P_{0}:(-0.13,0,0.5), P_{1}$ : $(-0.23,-0.40,-0.5), \quad P_{2}:(-0.60,-0.47,-0.5)$, $P_{3}:(-0.70,0,-0.5), \quad P_{4}:(-0.69,0.19,-0.41)$, $P_{5}:(-0.60,0.35,0.5)$ and finally it returns to $P_{0}$. Choosing $x_{0}=-0.1$ the trajectory returns to $x_{6}=-0.14$, which indicates that this closed trajectory is asymptotically stable.

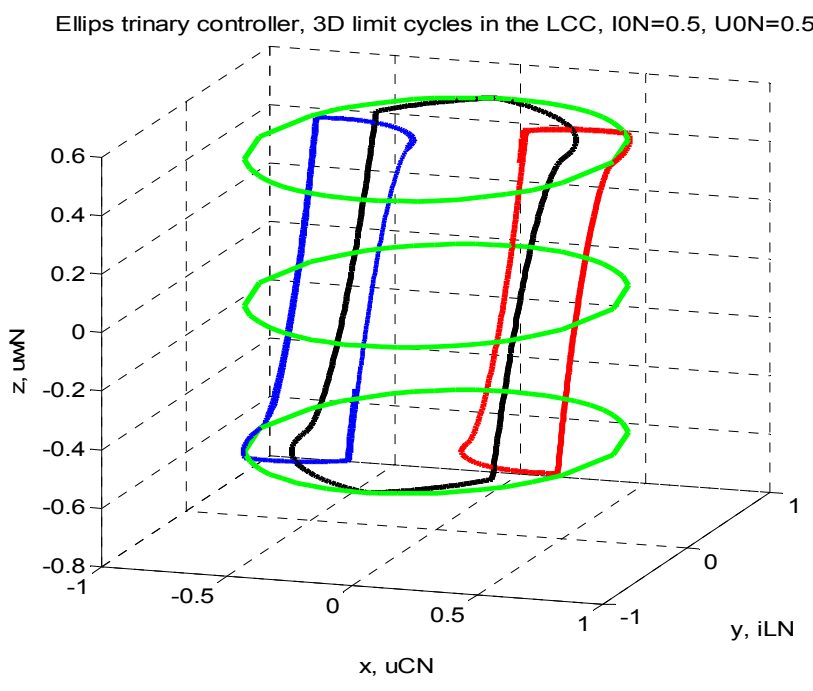

Fig. 4 Three different limit cycles exist for the controlled converter, one unstable limit cycle in the central part and two stable limit cycles.

\subsection{The Limit Cycle to the Right.}

Choosing a starting point at $P_{0}$, the trajectory intersects the cylinder shell on its way down instead at $P_{1}$. It intersects the bottom at $P_{2}$, the switching surface at $P_{3}$. It follows a plane up to the top with intersection at $P_{4}$, intersects the ellipse at $P_{5}$ and finally returns to the switching surface $y=0$ at $P_{6}$. Based on the same discussion as in previous sections there are closed trajectories through the points $P_{0}:(0.70,0,0.5)$, $P_{1}:(0.69,-0.21,0.40), \quad P_{2}: \quad(0.60,-0.39,-0.5)$, $P_{3}:(0.10,0,-0.5), P_{4}:(0.20,0.41,0.5), P_{5}:(0.59,0.50,0.5)$ and finally $P_{6}=P_{0}$ can be found. Choosing $x_{0}=0.8$ the trajectory returns to $x_{6}=0.70$, which indicates that this closed trajectory is asymptotically stable.

For a certain parameter setup, including $\mathrm{I}_{0 \mathrm{~N}}$ and $\mathrm{U}_{0 \mathrm{~N}}$, the system can reach one out of three different closed trajectories depending on the initial conditions. In Fig. 5 the projection of three trajectories onto the xy-plane is shown. One trajectory has initial values on the unstable closed trajectory. The other two have initial values close to them. It can be seen that one trajectory enters the left limit cycle and one the right limit cycle after some revolutions. 
As presented in the previous sections, the stable limit cycles of the proposed controller are asymmetrical. . The asymmetry of the state-trajectories, gives rise to a question of saturation of the transformer core. Another question that arises is if the controller can deliver the correct load current, $I_{0 N}$. In this report only the transformer saturation problem is addressed.

The circuit model, Fig. 1, shows a transformer connected to the output rectifier. The objective of the transformer is to adapt the input voltage of the circuit to the needed voltage supplied to the load. In the mathematical modelling of the circuit, the transformer is assumed to be ideal. Hence, it is considered being lossless, having no magnetizing current, and no voltage drop. This results in an infinitely high inductance in parallel to $\mathrm{C}_{\mathrm{W}}$ and a zero-inductance connected in series with L, see Fig. 1. These components do not have an impact of the operation of the circuit and is not represented in the mathematical model (2) and (3).

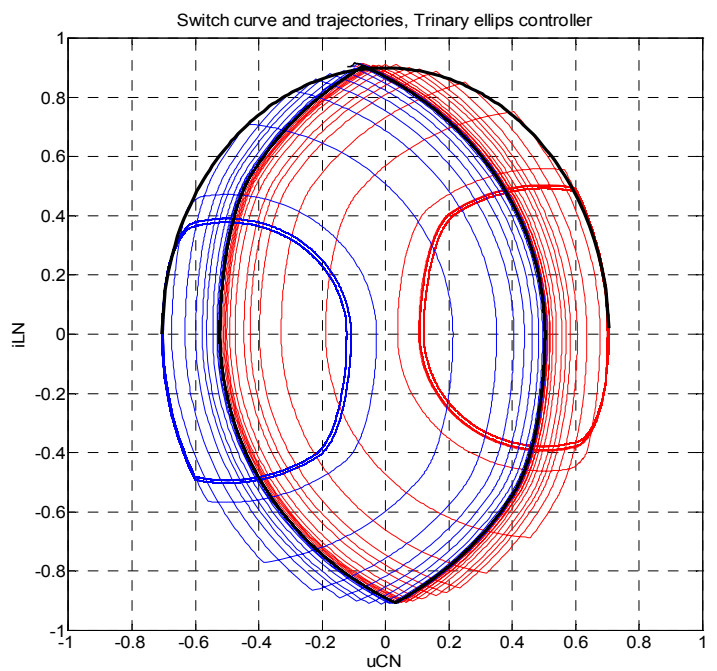

Fig. 5. Projection of transient trajectories and limit cycles onto the xy-plane. All initial values are close to $(-0.1,0.9,0)$ Two stable asymmetric limit cycles and one non stable limit cycle are can be seen together with the controller switch curves on the upper half plane.

When a real transformer is considered in the actual circuit, the influence of a finite magnetizing inductance has to be evaluated. The magnetizing current is given by $d i_{L} / d t=u_{C_{w}} / L_{m}$ where $\mathrm{L}_{\mathrm{m}}$ is the magnetizing inductance of the transformer. Considering the asymmetrical trajectories of the limit cycles it appears that there is a risk for transformer core saturation. If the time-average of $\mathrm{u}_{\mathrm{wN}}$ is not zero, this will result in very high magnetizing current which will drive the core into saturation.

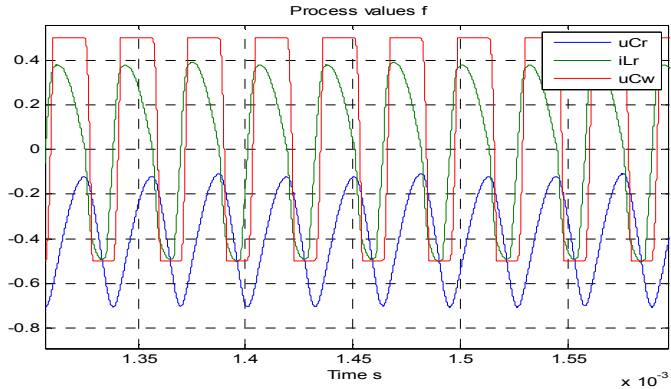

Fig. 6. Time diagram for the states $x(t), y(t)$ and $z(t)$, when the trajectory follow the left limit cycle.

Fig. 6 shows a time diagram of $i_{\mathrm{LN}}, \mathrm{u}_{\mathrm{CN}}$, and $\mathrm{u}_{\mathrm{wN}}$ in the time plane, where the red trace shows $u_{w N}$. It is noted that the amplitude of $\mathrm{u}_{\mathrm{wN}}$ is symmetrical but the time of the positive and negative half period differs. Thus, the time-average of $\mathrm{u}_{\mathrm{wN}}$ has a finite value, which is not zero. In Fig. 7 the straight line shows how the integration of $\mathrm{u}_{\mathrm{wN}}$ by time increases by time.

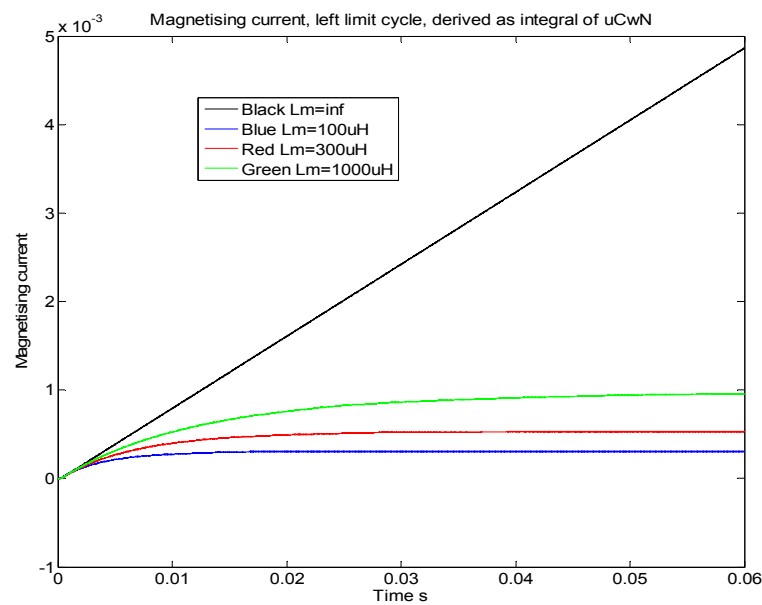

Fig. 7. Time diagram of the integration of $\mathrm{u}_{\mathrm{wN}}$ by time for different values of magnetizing inductances.

Two questions arise from the consideration of a non-ideal transformer: Will the magnetizing current have an impact on the limit cycles? Is it possible to avoid transformer saturation? In order to address these questions a set of simulations have been performed where a finite value of the magnetizing inductance has been introduced. The effect of this has been evaluated both in respect of the limit cycles and the transformer saturation. Including a finite magnetizing inductance and defining the new state $w=i_{L m N}=\frac{i_{L m}}{E} \sqrt{\frac{L}{C}}$, the system can be simulated as a fourth order system.

Fig. 7 also shows the integration of $u_{\mathrm{wN}}$ for different values of the magnetizing inductance, $0,1 \mathrm{mH}, 0,3 \mathrm{mH}, 1 \mathrm{mH}$. Fig. 8 shows the resulting magnetizing current when the magnetizing inductance is finite. Simulations show that including a magnetizing inductance does not significantly affect the limit cycle behaviour as presented in Fig. 5. 


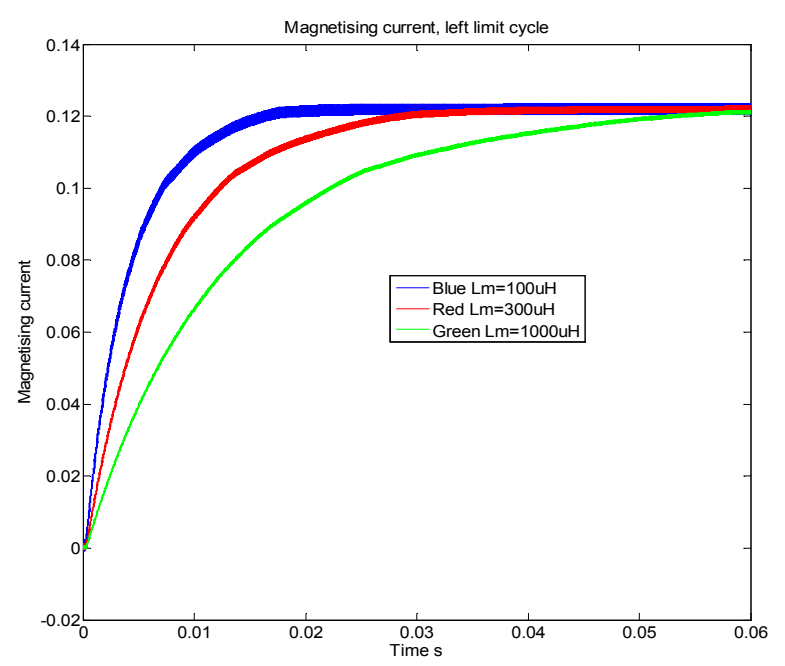

Fig. 8. Including the magnetizing current

When considering saturation of a transformer core, normally a blocking capacitor is connected in series with the primary winding. In the circuit analyzed in this paper, it will have no effect, as the origin of the DC-voltage component of $u_{w N}$ is the asymmetrical limit cycle. Any DC-voltage component from the source is blocked by the capacitor, C, of the resonant tank. From Fig. 8 it is observed that the resulting magnetizing current is limited and has the same value for the different values of the magnetizing inductance simulated. This indicates that the saturation issue caused by the asymmetries may be handled by the introduction of an air gap in the transformer core. The air gap will increase the energy storage capability of the transformer. The stored energy of an inductance is given by, $W=L i^{2} / 2$ which indicates that decreasing the inductance while keeping the current constant, will decrease the stored energy. The energy storage in a magnetic field can be calculated by the integration of the energy density over the volume, $\mathrm{V}$, of the field,

$$
W=\int_{V} \frac{d W}{d V}
$$

where the energy density is given by,

$$
\frac{d W}{d V}=\frac{B^{2}}{2 u u_{0}} .
$$

$\mathrm{B}$ is the flux density, $u$ is the relative permeability of the material and $u_{0}$ is the permeability of vacuum. Equations (6) and (7) yield that the introduction of a material with a low value of $\mathrm{u}, \mathrm{u}_{\mathrm{air}}=1$, will increase the energy storage capability of the transformer at a given value of $\mathrm{B}$. Thus, avoiding the saturation of the core. The drawback of an air gap is a reduction of the magnetizing inductance resulting in an increased AC component of the magnetizing current. The magnetizing- current flows in a parallel path compared to the load current. Thus, an increase of the magnetizing current will normally have the negative effect of increasing the total losses of the circuit.

\section{CONCLUSION}

An industrially applied LCC power converter is modelled as a hybrid system. It is found that the hybrid system given a parameter set up and different initial conditions has three different limit cycles. One almost symmetrical limit cycle and two asymmetric limit cycles. The two asymmetric limit cycles are stable while the third one is not. It is also noted that the energy supplied to the load is lower for the stable ones compared to the unstable.

Effects of the asymmetric limit cycles are considered. It is found that the asymmetry of a limit cycle will generate a high magnetising current possibly driving the transformer core into saturation. The saturation problem can be met with introducing an air gap in the transformer to the cost of introducing higher losses.

It is judged that when introducing switched controllers in industrial applications the solution set has to be carefully analysed. The solution set of hybrid system can be very rich.

\section{REFERENCES}

Chen H., Sng E., Tseng K.-J. Generalized Optimal Trajectory Control for Closed Loop Control of Series-Parallel Resonant Converter. IEEE Transactions on Power Electronics, vol. 21, no. 5, Sept. 2006.

Flieller P.R.D., Louis J. Computation and stability of limit cycles in hybrid systems, Nonlinear Analysis 64 (2006) 352-367.

Hultgren A., Ranstad P., Lenells M. Ingelbrant P. and Nilsson M., "Switched Controllers Applied to a LCC Conv.", Proc. of NORpie/2002, IEEE and Pels, Stockholm, Sweden, Aug. 12-14 2002.

Hultgren A. and Lenells M. Stability of an switched Hamiltonian observer applied to an SLR converter. Proceedings of IEEE PESC 2004 June 2004, Aachen, Germany.

Hultgren A. and Lenells M. Stability of a nonlinear Hamiltonian Observer Applied to a General Electrical Network. 43rd IEEE Conf. on Decision and Control, The Atlantis Paradise Island, The Bahamas, ISBN 0-78038683-3, pp 4806-4811, December 14-17, 2004.

Melin J. and Hultgren A. A limit cycle of a resonant converter. Proceedings of IFAC ADHS conference June 2003, St. Malo, France, 2003.

Melin J. and Hultgren A. On conditions for regularity of solutions for a piecewise linear system. Journal of Nonlinear Analysis: Hybrid Systems and Applications, 65(12):2277-2301, 2006.

Melin J., Hultgren A. and Lindström T. Two Types of Limit Cycles of a Resonant Converter Modelled by a Threedimensional system. Journal of Nonlinear Analysis: Hybrid Systems and Applications, 2 (2008) 1275-1286 Elsevier Inc. 2008.

Orlov Y.V. Discontinuous Systems. Springer 2009.

Oruganti R. and Lee F.C. Resonant power Processors: Part 2-Methods of Control, IEEE-IAS ann. meet 1984.

Rossetto L. A simple Control Technique for Series Resonant Converters. IEEE Transactions on Power Electronics, vol. 11, no. 4, pp 554-560, 1996.

Van der Schaft A. and Schumacher H. An introduction to Hybrid Dynamical Systems, Springer, 2000. 